\title{
Dynamic routing based on a single parameter: Link Congestion Probability
}

\author{
J.L. Marzo*, J. Domingo**,R. Fabregat ${ }^{*}$, J. Sole** \\ * Universitat de Girona. EPS. Avda. Lluis Santalo s/n (17071) Girona \\ (Spain). Tel: +34 72 418484.Fax +34 72 418399.e-mail marzo@ei.udg.es \\ ** Universitat Politècnica de Catalunya. DAC. Campus Nord. \\ Barcelona (Spain). Tel.: +34 3 4016982. Fax +34 34017055.
}

\begin{abstract}
In this paper we present a dynamic routing algorithm with alternative paths for call establishment on ATM Networks based on anticipated calculations. The routing decision criteria basically depends on the type of source of the new connection and the current network load supported by the network. We suggest an algorithm based on the Probability of Congestion in the link as an optimal control parameter for routing decision. The calculation of the Probability of Congestion is based on the Convolution Approach.

The objectives of our suggestion are: to obtain a fast connection setup with the routing algorithm, to define an optimal Routing Control Parameter, to maximize network resources utilization and to minimize call blocking probabilities. In order to obtain fast connection setup when a new connection demand arrives, each node already has a pre-computed Probability of Congestion table for each type of source and for each output Virtual Path. To obtain the improvement of resource utilization we suggest a dynamic routing scheme with alternative routes. The method proposed for the selection of the route is based on the well-known Least Loaded Routing (LLR) algorithms used in circuit switched networks. Different routing algorithms are compared when changing the criteria by the selection of available alternative routes and when changing the choice of alternative route.
\end{abstract}

Keywords

ATM, Dynamic Routing, Bandwidth Allocation, Connection Admission Control, Convolution Approach, Probability of Congestion, Individual Cell Loss Probability, QOS

\footnotetext{
${ }^{1}$ This work has been supported by CICYT (Spanish Education Ministry) under contract TIC92-1289-PB.
} 


\section{INTRODUCTION}

Network Provisioning (NP) is the set of the long-term control actions that determine the physical quantities of the resources to be placed in the network. Given the network resources by a suitable NP, Network Resource Management (NRM) is the set of control functions related to establish VPs and to allocate bandwidth, which are performed by the network in order to optimize performance and utilization objectives, namely to provide the required Quality of Service (QOS) and to maximize utilization, with simple Connection Admission Control (CAC) and Routing procedures. The drawback of many existing routing algorithms is the lack of cooperation between congestion control and routing.

\section{Bandwidth Allocation}

The basic objective of a bandwidth management and traffic control strategy in Asynchronous Transfer Mode (ATM) network is to allow for high utilization of network resources (transmission bandwidth, buffer space, etc.), while sustaining an acceptable QOS for all connections. Several network traffic control functions, such as congestion control and routing, depend on the characterization of the bandwidth required for each individual connection and the resulting load on network links and buffer utilization.

Some authors assign for each source an equivalent bandwidth that reflects its characteristics. Usually this involves a reduction in the complexity, and therefore a reduction of the accuracy of the evaluation models. In (Guerin, 1991) an approach is proposed that combines two basic approximated models to study Bandwidth Allocation: the fluid flow model and the stationary model. By the second model, the convolution approach is the most accurate method used in bandwidth allocation when a priori traffic estimation is considered. One important aspect of Bandwidth Allocation is CAC.

\section{Connection Admission Control}

CAC addresses a set of actions taken by the network at the connection setup phase (or during the connection re-negotiation phase) in order to establish whether a new connection can be accepted or rejected. The exact evaluation of the offered load in ATM Networks can be calculated by the convolution approach. But it has a considerable computation cost and a high number of accumulated calculations. Nevertheless, in critical near-congestion situations, the convolution is an algorithm that gives enough accuracy in order to exploit a maximum multiplexing gain. In (Marzo, 1993) and (Fabregat [b] 1994) we propose the use of the New Convolution Approach (NCA). In this case the NCA offers a possible fast evaluation without accuracy loss. The adopted CAC policy has a strong influence on the routing performance.

\section{Routing in ATM Networks}

It consists on assigning a path ${ }^{2}$ to the incoming connection demands during the call setup. For each new call $^{3}$ the network must select a path that has sufficient bandwidth available to support the new connection and to guarantee the required Quality of Service (QOS). Otherwise, the new call will be blocked (or rejected). Cells of the connection are transferred along the assigned route. The traffic produces random variations in the link occupancies in the

\footnotetext{
2 Throughout this paper the terms "path" and "route" are interchangeable

3 Throughout this paper the terms "call", "connection demand" and "Virtual Circuit" are interchangeable.
} 
network. The aim of a routing scheme is to induce, as fas as to the possible, these random variations in the pattern of link occupancies to maximize network resources utilization and to minimize network blocking (the probability of an arriving call finding no suitable idle path for its connection). The routing policy attempts to find the path that causes the least damage to the network.

\section{Alternative Routing}

Dynamic routing gives a better chance of success to an individual call by increasing the number of ways it can traverse the network. When a new call requests establishment between a pair of switches, it is possible to consider routing schemes in VP networks which always offer the direct VP first; then this path/route will be used with priority. If a call is blocked on the direct VP, the call is setup on an allowable alternative route. Otherwise, the call is blocked. The routing algorithms differ in how they choose from among the set of allowable alternative routes. By providing alternative paths, ATM networks will be able to achieve lower connection blocking probabilities and higher network throughput, while still satisfying various QOS requirements through the rigorous application of CAC. The blocking probability can be decreased by using alternative paths (Spiegel, 1994). Several studies exclude alternative routes that consist of more than two VPs. Thus, every pair of terminals has an associated set of routes, where each route consists of one direct VP, or two VPs as alternative routes (Bahk, 1994), (Gupta, 1993), (Ohta, 1992), (Mase, 1991), (Kolarov, 1994), (Oser, 1994). In (Lin, 1993) and (Cheng, 1994) the effects of limiting the maximum number of physical links for each path are discussed. It is shown in (Ahn, 1994) that providing even as few as 2 or 3 multiple paths per source-destination pair will result in a marked decrease in call blocking probability.

The two VP constraint simplifies routing implementation and ensures loop-free routing. Removing the restriction to two VPs, of course, allows a wider choice of alternative routes. One effect of this is that it gives more flexibility in routing and, thus, tends to reduce the blocking probability. Another effect is the reduction of the effective capacities of the physical links. This effect tends to increase the blocking probabilities. This result implies that the effect of the increase of routing flexibility is overweighed by the effective capacity reduction. However, in general, there is a price to be paid for the use of multiple VP for a single call, viz., the fact that it constitutes an inefficient use of network resources to tie up several VP for the sake of a single call, while those VPs might be used to complete several separated calls (Krishnan, 1994), (Lin, 1993). Moreover, the cost of routing the call over a multiple-VP route is the sum of costs of routing the call over its constituent VPs (Kolarov, 1994).

When a call is to be routed on an alternative path consisting of two VPs, we again encounter the following problems: how to characterize the traffic in a VC as it travels along the alternative path and how to assign the end-to-end QOS requirement to each link of the alternative path; note that for a call that is alternatively routed, cell loss, cell delay, etc., can occur at either of the two VPs along the route. Two classes of QOS, one for direct calls and one for alternative calls, need to be established on a VP that contains alternative routed calls. When a VC is routed on an alternative path, it requires a more stringent QOS at each VP on this alternative path, since QOS is an end-to-end measure and multiple VPs are now the endto-end path.

\section{Background Studies}

In (Gupta, 1993), two properties, heterogeneous sources (yes or no) and statistical multiplexing inside the network (yes or no), lead to four classes of ATM networks. When all sources have the 
same traffic characteristics and QOS requirements (homogeneous sources) and the VCs are statistically multiplexed, various Least Loaded Routing (LLR) algorithms are proposed and developed to reduce the network call blocking probability. The work assumes that the QOS requirement only involves cell loss, although a previous study by the same author (Gupta, 1992) examined QOS requirements based on delay as well as cell losses. The interest in developing dynamic routing algorithms which incorporate the diverse nature of calls is addressed in (Gupta, 1994).

Other works seek to route each call in such a way that it minimizes the risk of blocking future calls, responding to the current state of the network on the basis of certain assumptions about future traffic demands. The network state can be characterized as a Markov Decision Process (MDP). When a call reaches a given state, out of the possible measures, the one that leads to the state with the smallest cost should be chosen (Hwang, 1994).

In (Oser, 1994) an optimal control parameter, $\beta$, is developed by optimal control theory for ATM networks. The parameter is related to link utilization, buffer occupancy, system throughput and cell rejection characteristics. This single parameter is a function of instantaneous cell loss, buffer occupancy, utilization and user requirements. The parameter $\beta$ is applied to route selection and admission control.

In (Fabregat, 1994) we propose anticipated calculations by CAC and adaptive routing with a pre-evaluated scheme. The behavior on a link of the selected route using the pre-evaluated scheme is verified by comparison to ordinary and optimum evaluation. Incoming connection and disconnection demands are used under different traffic characteristics assumptions.

This paper is organized as follows. Section 2 gives a detailed explanation of the proposed routing scheme based on convolution approach. Section 3 deals with simulation models and results, followed by conclusions and further works in Section 4.

\section{DYNAMIC ROUTING ALGORITHM BASED ON THE CONGESTION PROBABILITY}

The objectives of our proposal are: to obtain a fast connection setup with the routing algorithm, to define an optimal Routing Control Parameter, to maximize network resources utilization and to minimize call blocking probabilities. To obtain an accurate fast response with the routing algorithm, pre-evaluated calculations of the CAC are needed. To obtain the improvement of resource utilization we propose a dynamic routing scheme with alternative routes. The Probability of Congestion (PC) in the VP (link) is the control parameter selected for routing decision.

QOS is normally expressed in terms of CLP, cell delay variation and maximum cell delay, in which the maximum cell delay can easily be imposed by having a finite buffer to limit the maximum queue length and consequently the maximum delay. Therefore, some authors: (Atkinson, 1994), (Bahk, 1994), (Chan , 1994), (Gupta, 1993), choose the CLP as the QOS measure for routing decision.

If statistical multiplexing is considered it may happen that the bandwidth required by the accepted connections exceeds the capacity of the link, and its probability is evaluated with the Probability of Congestion (PC) on the link. For this reason the PC is a value of the link utilization. The PC, the Total CLP and the Individual CLP can be calculated using the New 
Convolution Approach (NCA). In this paper the performance criteria by CAC is Individual CLP requeriments and the PC was applied to route selection.

\section{Network Model}

Given a network topology, a capacity for each physical link and a traffic requirement for each origin-destination pair, a Virtual Path Network embedded in the original network can be defined. A Virtual Path is an information transport path that can be viewed as a single logical direct link between two nodes (source and destination) with a capacity assigned.

The use of VPs in ATM networks reduces the call setup delay, simplifies the hardware in the transit nodes and provides simple virtual circuit admission control. However, it also reduces the degree of capacity sharing and, thus, increases the call blocking rate for most cases. In a network using VPs, two levels of statistical multiplexing are possible. VCs are statistically multiplexed onto VPs and these VPs can be statistically multiplexed onto network links. If both these levels of multiplexing are employed (with due regard to Quality of Service requirements), the capacity required in the network will again be a minimum (Burgin, 1991). There is clearly a trade-off between resources spent on management and carried traffic. We assume that only the VCs statistically multiplexed onto the VP are considered.

The system of virtual paths in an ATM network can be established based on estimated traffic demands. VPN establishment is beyond the scope of this paper. We consider ATM networks where only one VP is established between every origin node of the connection demand and the corresponding destination. This requirement can seem very restricted, yet it is not so if we take into account that we are not concerned with the problem of routing between nodes of different networks (internetworking), but the fact that the origin-destination pair are inside the same network. We assume a semi-permanent VPN topology because it can be updated periodically if changing traffic demands make it necessary.

We assume that the time interval between two VPN topology changes is significantly larger than the call setup time. Under this assumption, routing of virtual circuits can be performed as if the topology of the VP networks were fixed. Several VPs may share a physical link with each VP having resources assigned. We suppose that each VP has a dedicated buffer and its bandwidth is fixed and constant. The sum of the VP capacities is required to be less than the capacity of the link. The set of possible routes consists of one direct VP, or two VPs, as alternative routes, with the QOS requirements divided between the two VPs. The VPN is referred to as a heterogenous VPN in (Gupta, 1993) because at least one VP supports calls that have different traffic characteristics and/or QOS requirements.

\section{Traffic Model}

The question of how to multiplex two or more diverse traffic classes while providing different QOS requirements is a very complicated problem. Each connection demand is characterized by the traffic types, the required QOS, the origin-destination pair, the interarrival time and the duration of the connection (holding time).

There are ST types of traffic modelled by General Modulated Deterministic Process (GMDP) explained in (Race 1022). This model describes the behavior of a traffic source at cell and burst level. It consists of a finite number of states. In each state, cells are sent at a costant rate during a general distributed sojourn time. After the sojourn time has ended the process enters another state with a certain probability. In the GMDP model several traffic types are defined by varying the number of states, the bit rate of the state and its corresponding 
probability. The arrival connection of traffic type $\mathbf{j}$ is assumed to follow a Poisson process with rate $\lambda_{\mathbf{j}}$. The connection holding time is assumed to have an independent and exponentially distributed function with mean $1 / \mu_{\mathrm{j}}$. We assume that when a call is denied access to the network, it does not retry and is considered lost.

\subsection{Connection Setup Scheme}

To obtain an accurate fast response with the routing algorithm we propose to have the CAC calculations before the new connection demand arrives. Different evaluation schemes can be considered:

\section{On-Line Pre-Evaluation Scheme}

The on-line pre-evaluation scheme consists of updating dynamically the status of the output VP when the CAC-evaluation subsystem is idle. For each type of source a new virtual connection must be considered. This process allows the system to know whether or not it is possible to allocate this hypothetical new call. Using the pre-evaluated response table, it is possible to know beforehand if this selected output VP has the sufficient bandwidth available to support one new connection of a given type.

For each output VP on the node a response vector $\mathbf{R}(\mathbf{v p )}$ is used to store results: $\mathbf{R}(v p)=\mathbf{R}_{0}, \mathbf{R}_{1}, \ldots, \mathbf{R}_{\mathbf{j}}, \ldots, \mathbf{R}_{\mathrm{ST}-1}$, where $\mathbf{S T}$ is the number of possible Source Types. $\mathbf{R} \mathbf{j}$ can be:

- YES, when a new j-type connection can be accepted because all Individual Cell Loss Probability (Individual CLP) calculated are smaller than the Individual CLP requirements.

- NO, when a new j-type connection cannot be accepted because some of the Individual CLP calculated are larger than the Individual CLP requirements.

- UNKNOWN, when the CAC-evaluation subsystem has not yet evaluated a new j-type connection.

The following background process is run to fill all the elements of the response vector $R(v p)$ with the value YES or NO. The elements that are equal to UNKNOWN are the only ones evaluated. When an incoming call or a disconnection arrives the CAC-evaluation subsystem interrupts the background process and processes the new event. The disconnection process consists of the re-evaluation of all the elements of the response vector $R(v p)$ that are equal to NO. After the call termination the responses of $\mathbf{R}$ equal to $\mathrm{NO}$ are transformed into UNKNOWN in order to be re-evaluated. Note that YES responses do not need to be updated because the system load has decreased. These responses will continue to be equal to YES. However, the connection setup process consists of a re-evaluation of all the elements of R(vp) that are equal to YES, so that those elements equal to YES are transformed into UNKNOWN. In this case the NO responses do not vary because the system load has increased.

\section{Storing the past}

As the probability of congestion only depends on the current traffic demands and the value of the VP capacity assigned to the VP and as we suppose that the VPN is semi-permanent, often the same situations may be calculated. Therefore, an immediate response can be obtained if all 
evaluated results are stored. Moreover, the status of the output VP exhibits a locality, meaning that the status of the system does not suffer drastic changes and for a relatively long period of time the number of connections on the VP are only a relatively small fraction of all possible cases. When the entire possible status is in the memory, the CAC-evaluation subsystem will offer a response without making any calculations. If there is sufficient memory to hold the entire possible status, all the calculations can have been made previously (Off-line evaluations). If the available memory is too small to hold the entire possible status, the CAC-evaluation subsystem will make many calculations.

\subsection{Alternative Routes}

To obtain the improvement of resource utilization we propose a dynamic routing scheme with alternative routes. Each node must maintain a table specifying the $\mathrm{PC}$ of each possible outputVP and destination pair node. Different routing algorithms can be implemented by changing the choice from the set of alternative routes. One possibility should choose the alternative route so that the congestion on the VP is minimized. The call is blocked if no alternative routes are available. When a demand is accepted or when there is a disconnection, the Probability of Congestion stored in the adaptive routing tables must be updated according to the new state.

By providing alternative routes, the direct route will be able to achieve lower connection blocking probabilities and higher network throughput. However, the use of alternative routes entails the utilization of other VPs and, therefore, a reduction of their capacity and an increase of their blocking probabilities. Link congestion can occur at any VP along the route and more stringent QOS requirements must be considered. Therefore, the call blocking probability increases, as because calls that can be accepted on these VPs along a direct route are rejected with the new QOS requirements. As two classes of QOS need to be established on a VP that contains alternatively routed calls, the CAC must calculate the PC for the two QOS.

\subsection{Routing Control Parameter}

As is show in the above section, there is a trade-off between the utilization of the VP and the cost-penalty when two VPs of an alternative route with a more stringent QOS are considered. Therefore, a Routing Control Parameter (RCP) is defined and the next routing algorithm is considered:

choose direct route if $\mathrm{PC}$ (direct route) $<1$

else out of all available alternative routes choose alternative route with min PC. else reject

where: One alternative route is available only if ( $\mathrm{PC}$ (first VP) $<\mathrm{RCP}$ and $\mathrm{PC}$ (second VP) $<$ RCP); and PC(alternative route) $=\mathrm{PC}$ (first VP) + PC(second VP).

Note that an alternative route is only considered if the PC on each VP of the alternative route is smaller than the choose RCP. When the Routing Control Parameter is used, some amount of bandwidth is reserved to direct traffic into a VP. This increases the probability of the direct routed calls being set up. When RCP is ignored, the cost per connection (number of VPs and physical links per connection) will be increased because carrying out a connection by an alternative route utilizes two VPs instead one direct VP. 


\section{PERFORMANCE OF ROUTING ALGORITHM}

In this section, we present computer simulations to illustrate the behavior of the routing algorithm proposed by using the PC as the Routing Control Parameter. Let us now consider routing-call admission control in ATM networks where a call may be setup via a number of VPs and nodes. The alternative route is limited to a maximum of two VPs. In this case, if the alternative route is selected, the new connection must be accepted in each VP with a more stringent QOS (Individual CLP / 2). Routing algorithms differ in how they choose from among the set of allowable alternative routes. We study three algorithms: Direct) only direct route, each call is allowed to use exactly one VP; 1 alternative) only one alternative route; and 2 alternatives) choose available alternative route which has the smallest PC.

\section{The simulation model}

The network chosen for simulations (figure 1) allows to study the performance of each routing algorithm when different loads and RCP values are considered. The link capacity of each links has been set to $50 \mathrm{Mbits} / \mathrm{s}$ in order to decrese simulation effort.

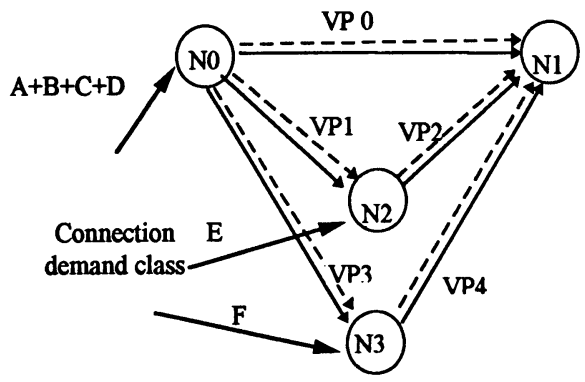

Figure 1 Virtual Path Network

Table 1 Demand Classes.

\begin{tabular}{|c|c|c|c|}
\hline $\begin{array}{c}\text { Conecction } \\
\text { demand } \\
\text { class }\end{array}$ & Orig. & Dest. & $\begin{array}{c}\text { Traffic } \\
\text { type }\end{array}$ \\
\hline A & N0 & N1 & 0 \\
\hline B & N0 & N1 & 1 \\
\hline C & N0 & N2 & 0 \\
\hline D & N0 & N3 & 0 \\
\hline E & N2 & N1 & 0 \\
\hline F & N3 & N1 & 0 \\
\hline
\end{tabular}

Traffic types are modelled by General Modulated Deterministic Process (GMDP) and grouped into traffic types which are shown in the table 2. Holding time and interarrival time have an exponential distribution. All time is expressed as a normalizated time units.

Table 2 Traffic types.

\begin{tabular}{|l|c|c|c|c|}
\hline traffic type & \multicolumn{2}{|c|}{0} & \multicolumn{2}{c|}{1} \\
\hline & rate & prob. & rate & prob. \\
\hline state 0 & 0 & 0,9 & 0 & 0,6 \\
\hline state 1 & \multicolumn{2}{|c|}{0,1} & 4 & 0,4 \\
\hline mean rate & \multicolumn{2}{|c|}{$0,4 \mathrm{Mbit} / \mathrm{s}$} & \multicolumn{1}{|c|}{$1,6 \mathrm{Mbit} / \mathrm{s}$} \\
\hline burstiness & \multicolumn{2}{|c|}{10} & \multicolumn{2}{|c|}{2,5} \\
\hline Ind. CLP & \multicolumn{2}{|c|}{$1.0 \mathrm{E}-5$} & \multicolumn{2}{|c|}{$1.0 \mathrm{E}-6$} \\
\hline (Ind.CLP)/2 & \multicolumn{2}{|c|}{$0.5 \mathrm{E}-5$} & $0.5 \mathrm{E}-6$ \\
\hline
\end{tabular}

Table 3 Routing table.

\begin{tabular}{|c|c|c|}
\hline $\begin{array}{c}\text { connection } \\
\text { demand }\end{array}$ & direct route & $\begin{array}{c}\text { set of allowable } \\
\text { alternative } \\
\text { routes }\end{array}$ \\
\hline A,B & VP0 & $\begin{array}{c}\{(\mathrm{VP} 1+\mathrm{VP} 2) \\
\text { (VP3+VP4) }\end{array}$ \\
\hline C & VP1 & -- \\
\hline D & VP3 & -- \\
\hline E & VP2 & -- \\
\hline F & VP4 & -- \\
\hline
\end{tabular}

The set of possible connection demands and available routes is shown in table 3. Connection classes C, D, E and F are used to load the other VP's. Note that, in table 1, the connection demand class $\mathrm{B}$ has a different traffic type, and it is used to perform heterogeneous traffic. 
Off Line evaluations: If the Probability of Congestion of all possible combinations has been precalculated before starting the routing simulation, the connection setup delay does not influence the results of the routing algorithms.

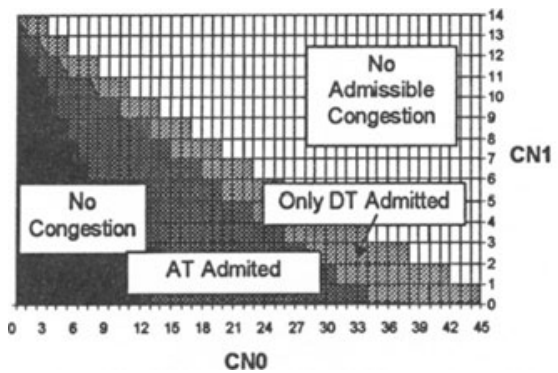

Figure $2 \mathrm{PC}$ by CAC using Individual CLP requeriments. PC maximum 2.67E-4

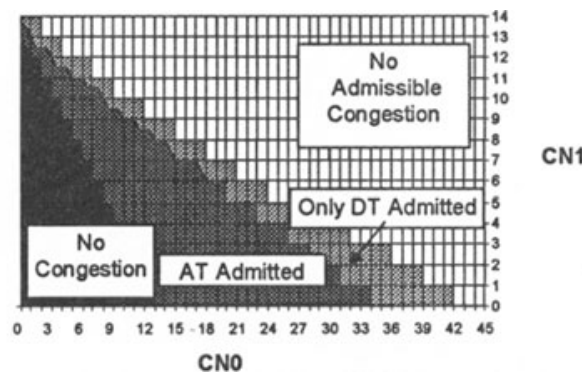

Figure 3 PC by CAC using (Individual CLP requeriments)/2. PC maximum 1.18E-4

In figures 2 and 3, we compare the maximum number of traffic type 0 and traffic type 1 connections that can be supported on a link with $50 \mathrm{Mbits} / \mathrm{s}$ of capacity under different Individual CLP requirements according to table 2. In figure 3 the Individual CLP requirements are more stringent. The RCP considered in both figures is $1.0 \mathrm{E}-5$.A point $(\mathrm{CN} 0, \mathrm{CN} 1)$ on the shadowed area can be interpreted as follows: CN0 traffic type 0 connections and $\mathrm{CN} 1$ traffic type 1 connections can be supported to guarantee the required QOS. On NoCongestion area the capacity required by $\mathrm{CNO}$ and $\mathrm{CN1}$ connections does not exceed the link capacity. On Alternative Traffic (AT) admited and only Direct Traffic (DT) admited areas the offered traffic exceeds the capacity of the link but the connections are accepted. Moreover, on AT admited area the PC is less than the RCP considered; that is, connections over VPs with alternative traffic are admitted. But on only DT admited area only direct traffic is considered. The border line between AT admited area and only DT admited area goes to No Admissible Congestion area if the RCP grows (1.0 E-4). But it goes to No Congestion area by smaller RCP (1.0 E-6). AT admited area only depends of the RCP, therefore they are the same in both figures. On No Admissible Congestion area, connections can not be supported with the Individual CLP requirements. The CAC and the routing algorithm must be applied using the figure 2 if only direct traffic is carried out on the VP, and using figure 3 if alternative traffic is carried out

\subsection{Simulations Results}

The performance of the network varies with the load of the system, with the routing algorithm and with the value of the RCP. Different system loads are obtained varying interarrival (ia) time: $i a_{A}=\{0.1,0.2,0.3\}$ and $i a_{C}=i a_{D}=i a_{E}=i a_{F}=\{0.10,0.15, \ldots, 0.50\}$. All connection demand classes have an holding time equal to 10 . The period of each simulation is equal to 1500 time units. The mean number of arrivals to the system depends on ia considered. For $\mathrm{ia}_{\mathrm{A}, \mathrm{C}, \mathrm{D}, \mathrm{E}, \mathrm{F}=0.1}$ there were 116000 arrivals. For $i a_{A}=0.1$ and $i a_{C, D, E, F}=0.5$ there were 33000 arrivals. Simulation is run 10 times for each set of parameters. For every simulations, the maximum magnitude of the $99 \%$ confidence intervals was within 0.02 of the value plotted. 
Figure 4 and 5 show the results of simulations for homogeneous traffic (connection demand $B$ is not considered) and $i a_{A}=0.1$. Two different values to RCP are considered: $1.0 \mathrm{E}-6$ and 1 (RCP ignored), as second value we can considered any value larger than the maximum PC admited. The legends show the results by different routing algorithms: Direct, 1 alternative (N0-N2-N1) and 2 alternative (N0-N2-N1 and N0-N3-N1).

Figures 4 shows Global Blocking Probability (GBP) for each Routing Algorithm by differents ia when RCP is ignored. While ia ${ }_{C, D, E, F}=0.1$ the GBP obtained by different routing algorithm are similar because the whole network is heavily loaded so the connection can not be accepted on alternative routes. When ia $\mathrm{C}_{C, D, F}<0.30$ and alternative routing algorithm is used, the GBP increases. The Blocking Probability of alternative VP increases because they are loaded with other connection demand. This occurs because traffic of connection demand $A$ rejected on VP0 is accepted by alternative routes. When iac,D,E,F $>0.35$ the other VP are lightly loaded, so they can accept the new traffic perfectly and the GBP decreases.

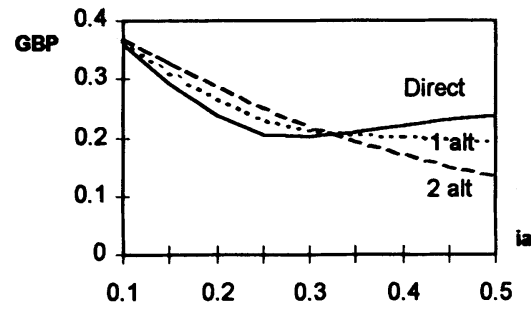

Figure $4 \mathrm{RCP}=1$ (RCP ignored)

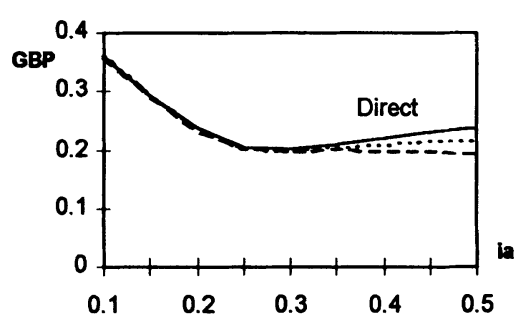

Figure $5 \mathrm{RCP}=1.0 \mathrm{E}-6$

Figures 5 shows GBP for each Routing Algorithm by differents ia $\mathrm{C}, \mathrm{D}, \mathrm{E}, \mathrm{F}_{\mathrm{F}}$ when RCP is 1.0E-6. When iac,D,E,F $<0.30$, the GBP of alternative algorithm decreases and it is very similar to the value obtained by Direct algorithm. When ia ${ }_{C, D, E, F}>0.30$, the GBP is greater than the value obtained when RCP is ignored, but it is always smaller than the GBP of the Direct algorithm. As we can see comparing figures 4 and 5, the GBP of the Direct algorithm was not affected by the use of RCP just because alternative routes are not considered.

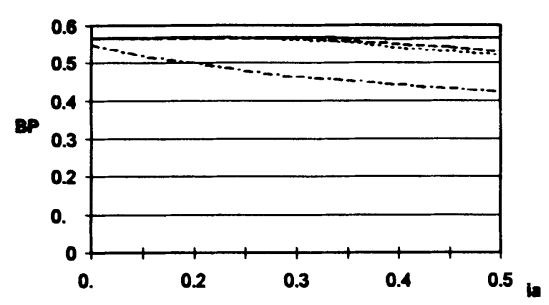

Figure 6 VPO BP (1 alternative)

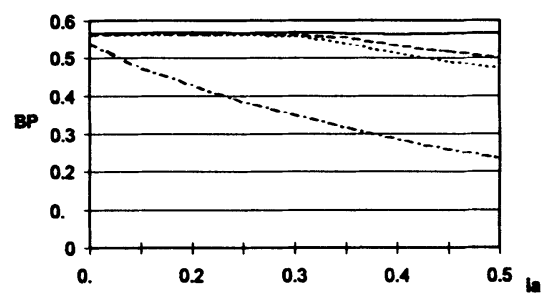

Figure 7 VP0 BP (2 alternatives)

- Direct $\quad----$ RCP=1E-06 …... RCP-SE-06 -...NORCP




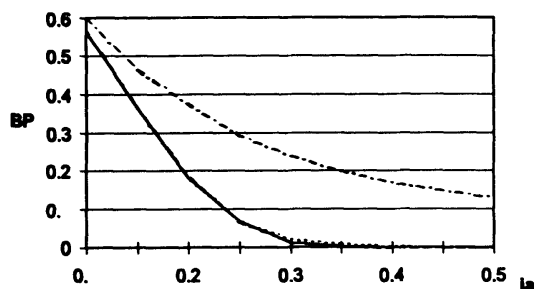

Figure 8 VP1 BP(1 alternative)

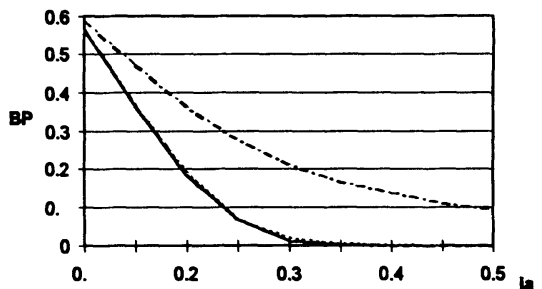

Figure 9 VP1 BP (2 alternatives)

Figures 6 and 7 show the Blocking Probability (BP) on VP0 for each RCP by differents ia $a_{C, D, E, F}$ when Routing Algorithm is 1-alternative (2-alternative respectively) and $i a_{A}=0.1$. Note that the BP of the Direct algorithm is show by compare the results. Figures 8 and 9 show BP on VP1. As expected, using alternative routes by ignoring the RCP the BP of VP0 decreases, but the BP of the alternative VPs used increases dramatically (figures 8 and 9).

A set of RCP values has been studied and the obtained results are similar to the shown values in above figures. The upper limit for RCP is not equal 1, in fact it is the maximum PC in the alternative path ( $\cong 1 E-4$, see figure 3 ).Finally, BP has a good behavior for all the network loads by using the RCP. When the network is loaded, up to iac,D,E,F $=0.3$, the BP is similar for different RCP values. When the network load decreases a BP improvement in the Direct route (VP0) is obtained, while the BP in the alternative path (VP1) remains practically invariable.Performance of routing algorithms with heterogeneous traffic are similar that which obtained with homogeneous traffic. Due to space limitations, this simulations graphs are omitted (Marzo, 1995).

\section{CONCLUSIONS AND FURTHER WORKS}

We suggest a dynamic routing algorithm with anticipated calculation based on the Probability of Congestion in the link as control parameter for routing decision. The Probability of Congestion is evaluated using the NCA, which permits fast calculation. The main objectives of our proposal are: to maximize network resources utilization and to minimize call blocking probabilities. Using alternative routes does not always decrease the blocking probability of the network. The Routing Control Parameter despite this drawback. One important aspect of future works is to determinate the best RCP based on the load of the network. Also, other allocation strategies and VPs statistically multiplexed onto the link will be studied in the future.

\section{Acknowledgements}

The authors thank Dr. Ian F. Akyildiz, Georgia Institute of Technology (Atlanta), for his many constructive observations and comments in relation with this work. 


\section{REFERENCES}

Ahn S. and others. "Virtual Path Layout Design On ATM Networks". INFOCOM'94

Atkinson. A."A Traffic Control Schem for Virtual Paths in an Asynchronous Transfer Mode Network". GLOBECOM'94

Bahk S. and El Zarki M. "Preventive Congestion Control based Routing in ATM Networks". ICC'94

Burgin J and. Dorman D. "Broadband ISDN Resource Management: The Role of Virtual Path". IEEE Communications Magazine. September 1991.

Chan J.H.S and.Tsang H.K "Bandwidth Allocation of Multiple QOS Classes in ATM Environment". INFOCOM'94.

Cheng K.T. and. Lin F.Y.S. "On the Joint Virtual Path Assignment and Virtual Circuit Routing Problem in ATM Networks". GLOBECOM'94

Fabregat R., Sole J, Marzo J.L. and Domingo J. "Bandwidth Allocation Based on Real Time Calculations Using the Convolution Approach". GLOBECOM'94.

Fabregat R., Sole J., Marzo J.L. and Domingo J. "Adaptive Routing Based on Real-Time Calculations Using the Convolution Approach.". EFOC'94.

Guerin R. and others. "Equivalent Capacity and its Application to Bandwidth Allocation in High-Speed Networks". IEEE Journal on Selected Areas in Communications, Vol 9, $\mathrm{N}^{\circ} 7$, September 1991.

Gupta S. and. Gandhi P.P. "Dynamic Routing in Multi-Class Non-Hierarchical Networks". ICC'94.

Gupta S., Ross K.W. and El Zarki M. "On Routing in ATM Networks". Modeling and Performance Evaluation of ATM Technology IFIP transactions (C-15).

Gupta S., Ross K.W. and El Zarki M. "Routing in Virtual Path Based ATM Networks". GLOBECOM'92.

Hwang R.H., Kurose J.E. and Towsley D. "MDP Routing in ATM Networks Using the Virtual Path Concept".INFOCOM'94.

Kolarov A. and Hui J. "Least Cost Routing in Multiple-Service Networks". INFOCOM'94.

Krishnan K.R. and Cardwell R.H. "Routing and Virtual-Path Design in ATM Networks". GLOBECOM'94.

Lin F.Y.S. and Yee J.R. "A Real-Time Distributed Routing and Admission Control Algorithm for ATM Networks". INFOCOM'93.

Marzo J.L, Fabregat R., Domingo J. and Sole J. "Fast Calculation of the CAC Convolution Algorithm using the Multinomial Distribution Function". Tenth UK Teletraffic Symp. 1993.

Marzo J.L., Domingo J., Fabregat R. and Sole J. "Dynamic routing based on a single parameter: Link Congestion Probability". Techical Report 95/001-EI. UdG. 1995

Mase K. and Shioda S. "Real-Time Network Management for ATM Networks". ITC-13.1991

Ohta S. andSato K.I. "Dynamic Bandwidth Control of the Virtual Path in an Asynchronous Transfer Mode Network". IEEE Transaction on Communications, Vol. 40, n. 7, July 1992.

Oser T.M., Gu X. and Vaman D. "Routing with Admission Control in ATM Networks". GLOBECOM'94.

RACE 1022. "Updated Results of Traffic Simulation of the Policing Experiment". Technology for ATD. December 1990.

Spiegel E.M. and Murase T. "An Alternate Path Routing Scheme Supporting QOS and Fast Connection Setup in ATM Networks". GLOBECOM'94. 